\title{
Dosis y frecuencia de biofertilizantes en Cucumis sativus L. Municipio de Siuna RACCN Nicaragua
}

\author{
Biofertilizers dosage and frequency in Cucumis sativus L. Siuna Municipality RACCN Nicaragua
}

\author{
Jamill Castillo Martínez' \\ Oscar Montalván Castellón²
}

\section{Resumen}

La aplicación de abonos orgánicos en la producción agrícola, ha constituido especial importancia desde la percepción de calidad, inocuidad y medio ambiente, en el municipio de Siuna, ya que se han realizado estudios sobre el efecto de los abonos orgánicos; sin embargo, no se han hecho estudios en la zona que evalúen las cantidades y frecuencias de los fertilizantes orgánicos especialmente los foliares.

Esta investigación evalúa los efectos de las dosis y frecuencias de aplicación de biofertilizante en el cultivo de Cucumis sativa L, desde su crecimiento, desarrollo y productividad, con la finalidad de evaluar con más detalle el comportamiento de cultivo, y poder recomendar de manera confiable cómo se pueden obtener máximos rendimientos a menores costos y sin afectar el medio ambiente. Es una investigación experimental en el nivel explicativa, se utilizó un diseño completamente aleatorio con tres replicas y un modelo estadístico donde se analizaron cada uno de los tratamientos.

Los principales resultados son: El crecimiento y desarrollo en el cultivo del pepino son similares en los diferentes niveles de concentración y aplicación del fertilizante, hay una mejor respuesta a una menor frecuencia y mayor dosis de aplicación del biofertilizante en el número de flores producidas por plantas y la producción de pepino es mayor con menor frecuencias y mayores concentraciones de fertilizante, y que por lo tanto también son más rentables.

Palabras clave: productividad; Inocuidad; crecimiento y desarrollo; rentabilidad.

\section{Abstract}

The application of organic fertilizers in agricultural production has been of special importance from the perception of quality, innocuousness and the environment, in Siuna municipality, since studies have been carried out on the effect of organic fertilizers; however, there have been no studies in the area that evaluate the quantities and frequencies of organic fertilizers, especially the foliar ones.

This investigation evaluates the effects of the biofertilizer application in doses and frequencies in the cultivation of Cucumis sativa L, from its growth, development and productivity, in order to evaluate in more detail, the cultivation behavior, and to be able to recommend in a reliable way how maximum yields can be obtained at lower costs and without affecting the environment. It is an experimental research at the explanatory level, we used a completely randomized design with three replicas and a statistical model where each of the treatments was analyzed.

\footnotetext{
1 Máster en Docencia Universitaria, Coordinador del área de Ciencia, Tecnología y Medio Ambiente de la Universidad de las Regiones Autónomas de la Costa Caribe Nicaragüense-Recinto Universitario Las Minas. Correo: Jasmillc@yahoo.com; ORCID: https://orcid.org/0000-0002-3648-9165

2 Máster en Docencia Universitaria. Técnico del Instituto de Recursos, Medio Ambiente y Desarrollo Sostenible de la Universidad de las Regiones Autónomas de la Costa Caribe Nicaragüense-Recinto Universitario Las Minas. Correo electrónico montesiuna@yahoo.com; ORCID: https://orcid.org/0000-0003-2576-0367
} 
The main results are: The growth and development in the cultivation of the cucumber are similar in the different levels of concentration and application of the fertilizer, there is a better response to a lower frequency and higher application of the biofertilizer dose in the number of flowers produced by plants, and cucumber production is higher with lower frequencies and higher fertilizer concentrations, and therefore they are also more profitable.

Keywords: Productivity; Safety growth and development; cost effectiveness.

\section{Introducción}

La producción de hortalizas se ha convertido en los últimos años en la alternativa de mejorar la alimentación y la economía de las familias productoras en la zona rural, la cual ha sido influenciada por organizaciones no gubernamentales. En el grupo de las hortalizas el pepino es una de las más cultivadas por su rápida producción, tiene mayor resistencia a plagas, enfermedades y variaciones climáticas; sin embargo, este cultivo presenta producciones bajas por problemas de floración, el cual es determinado por desbalance entre las flores femeninas y masculinas, influenciado por problemas nutricionales y biológicos.

La deficiencia de nutrientes se explica porque lo suelos de nuestra región no satisfacen los requerimientos nutricionales del cultivo, y es de nuestro conocimiento que las plantas para cumplir su ciclo fisiológico vital necesitan de otros nutrientes menores que no poseen los fertilizantes inorgánicos, ni se encuentran en proporciones suficientes en el suelo ( $\mathrm{Mg}$., $\mathrm{Fe}, \mathrm{Mn}, \mathrm{Cu}, \mathrm{Ca}$,) generalmente se encuentran en las materias orgánicas, ya sea en residuos de cosechas, como pajas o en los excrementos de los animales (Restrepo, 1996).

Para suplir esta deficiencia se ha estado incursionando en la aplicación de abonos orgánicos tanto foliares como el suelo, como una fuente de macro y micro nutrientes para satisfacer todo el ciclo biológico del cultivo desde su germinación hasta su fructificación; no obstante, la utilización de los biofertilizantes se ha realizado de manera incontrolada y sin previa documentación.

Esta investigación es experimental con la finalidad de contribuir al mejoramiento de la producción del Cucumis sativa L. mediante la aplicación de abonos foliares (biofertilizantes) con diferentes concentraciones y frecuencia de aplicaciones, en miras de obtener los mejores resultados con menos costos de producción y de esta manera consolidar una respuesta técnica, sobre cuáles son las dosis y frecuencias de abono foliar para obtener óptimos rendimientos.

Para tal efecto el estudio se realizó con dos diferentes dosis y dos frecuencias de aplicación en un diseño bifactorial completamente al azar, con dos factores (dosis y frecuencias) y dos niveles para cada uno de los factores. Esta investigación sirve para que las organizaciones no gubernamentales y estatales que trabajan en ese ámbito, tengan una base técnica para el cultivo orgánico de esta hortaliza con rendimientos óptimos.

De la misma manera, esta investigación sirve para el proceso de acompañamiento y extensión social que URACCAN realiza en las comunidades rurales, así como para consolidar los conocimientos teórico-prácticos de los estudiantes para que tengan una base técnica sólida sobre situaciones propias de la región y puedan de esta manera tomar decisiones agroecológicas en el ámbito productivo.

\section{Revisión de literatura}

Investigaciones realizadas en el Cucumis sativa $L$. en donde se estudió la dosis más efectiva de Fitomas derivadas de la caña de azúcar, para lo cual se determinaron tres concentraciones y un testigo. El cual refleja que la utilización de Fitomas a razón de 0.2 1/ha incrementó el rendimiento en 45\%, mientras que 
con 0.4 1/ha solo lo hizo para $25 \%$ y con 0.7 1/ha representó un 28\%, respecto al testigo. (López, Montano, Agustín, Beltrán, Rodríguez Pérez \& Berto; 2002).

Similares resultados obtuvo Montano (1998) en estudios realizados en el cultivo de la caña de azúcar, con el empleo del producto se incrementaron los rendimientos en un $27 \%$ como promedio con ahorros de \$58.45/ha por concepto de fertilizantes convencionales. Vásquez (2012), en una investigación que realizó sobre la produccion de pepino con biofertilizantes presento resultados de hasta 3.27 Kilogramos por planta, un $25 \%$ más que la fertilización química, esto significa la posibilidad de sustituir los fertilizantes químicos por biofertilizantes lo que conllevaría al ahorro de divisas por este concepto. Montano (1998), ha logrado sustituir totalmente la fertilización de la caña de azúcar en experimentos en parcelas semi controladas, produciendo un incremento de $23 \%$ en el rendimiento agrícola.

Marín (2017), registró en una investigación sobre la productividad del pepino con vermicompost y biofetlizantes, que después de 40 días de la siembra hay difeferencias significativas en las variables de altura o largo de la guía, peso y longitud del fruto, siendo la solución nutritiva de Steiner y efluente de vermicompost los que presentan los mejores resultados, esto significa la eficiencoa de abonos organicos foliares en la produccion del pepino.

Blandón, Blandón y Fernández, (2014). Indicó en una investigación sobre evaluación de fertilización orgánica y química en el rendimiento del cultivo de pepino que en las variables de desarrollo en plantas de pepino (Cucumis sativus L.), existe diferencias estadísticas significativas entre los tratamientos, para las variables longitud de la guía principal con promedios en el tratamiento químico $82.24 \mathrm{~cm}$, Lombrihumus $73.54 \mathrm{~cm}$ y pulpa de café más ceniza con $67.85 \mathrm{~cm}$. Número de hojas por planta con promedios en el tratamiento químico 11.45 hojas, Lombrihumus 9.88 hojas y pulpa de café más ceniza 8.58 hojas. Número de guías el tratamiento químico 2.24 guías, Lombrihumus con 1.73 guías y pulpa de café más ceniza con 1.51 guías, en base al análisis estadísticos se acepta la hipótesis alternativa de que al menos uno de los tratamientos es estadísticamente diferente.

Las mismas autoras reflejan que en las variables de rendimiento del cultivo de pepino (Cucumis sativus L,) existe diferencias estadísticas significativas entre los tratamientos para las variables número de frutos en el tratamiento químico con promedios de 3.41 frutos, Lombrihumus 2.54 frutos y pulpa de café más ceniza con 2.41 frutos. Longitud del fruto en el tratamiento químico con promedios de $16.08 \mathrm{~cm}$ pulpa de café más ceniza con 13,29 cm lombrihumus 13,26 cm.

En la variable diámetro del fruto; no se obtuvieron diferencias significativas entre tratamientos. En base a los análisis estadísticos se acepta la hipótesis alternativa de que al menos uno de los tratamientos es estadísticamente diferente, con excepción del diámetro del fruto. En relación costo-beneficio de la producción de pepino (Cucumis sativus L.) el tratamiento que presentó mayor costo de producción fue el tratamiento Lombrihumus $C \$ 1,052.05$, seguido el tratamiento químico con $C \$ 973.45$, Pulpa de café más ceniza con $C \$ 900.05$ y menor costo el testigo con $C \$ 847.55$. El tratamiento que presentó mayor beneficio fue el tratamiento químico con una relación de 1:2, seguido pulpa de café más ceniza con 1:1.52, en menor beneficio el Lombrihumus con 1:1.38 seguido el testigo con 1:1.09. (Blandón, Blandón, \& Fernández, 2014).

Estudios realizados en Argentina con abonos orgánicos en el cultivo del pepino principalmente con dosis de Lombrihumus definen que los mejores resultados para este cultivo son de 100 toneladas de Lombrihumus por hectárea donde registran significancia estadística del Lombrihumus sobre el inoculante en las variables de altura de la planta, área foliar y peso (Cracogna, Fogar, Iglesias y Verón, 2001).

En el 2000 se realizó un estudio sobre la producción de rebrote de arroz con la aplicación de biofertilizantes donde se utilizaron concentraciones de una media por cada 20 litros de agua y una frecuencia de aplicación de tres días, en donde se obtuvieron resultados positivos para este cultivo principalmente en la consistencia del grano (Castillo, Montalván, 2000). 
Otros estudios con resultados positivos fueron: establecimiento de maíz con aplicación de abonos orgánicos (Artola, Murillo, 2000 ), evaluación de variedades de arroz( Rodríguez, 2001) y evaluación de variedades de frijoles con aplicaciones de biofertilizantes (Ibarra, 2003), todos estos experimentos dieron resultados superiores en producción, comparados con las parcelas testigos sin fertilizantes, demostrando que para incrementar los rendimientos es necesario la aplicación de algún suplemento nutricional al suelo.

\section{Materiales y métodos}

El área de estudio se encuentra a 20 kilómetros al noroeste del municipio de Siuna, en la comunidad de El Hormiguero, específicamente en la finca experimental de URACCAN-Las Minas, la temperatura promedio es de $27^{\circ} \mathrm{C}$ con una mínima de $20^{\circ} \mathrm{C}$ y una máxima de $34^{\circ} \mathrm{C}$, con una humedad relativa de 35 a $80 \%$, con un régimen pluviométrico de $1500-3000 \mathrm{~mm}$ anual, siendo los meses de mayo a diciembre los de mayor lluvia, el suelo que predomina es del tipo aluvial diferenciado, producto de diferentes niveles de sedimentación con profundidad efectiva de más de 90 centímetros. Es un estudio cuantitativo, experimental con un diseño y análisis estadístico de diseño completamente al azar. Cuyo modelo estadístico es:

$Y i j=\mu+\tau i+\varepsilon i j$

Donde:

Yij observación del tratamiento i en la parcela j

ti efecto del tratamiento i

عij término de error aleatorio asociado a la observación Yij

Los tratamientos utilizados fueron distribuidos de la siguiente manera; $\mathrm{T}_{1}=\mathrm{F}_{1} \mathrm{D}_{1} ; \mathrm{T}_{2}=\mathrm{F}_{1} \mathrm{D}_{2} ; \mathrm{T}_{3}=$ $\mathrm{F}_{2} \mathrm{D}_{1} ; \mathrm{T}_{4}=\mathrm{F}_{2} \mathrm{D}_{2}$, en donde $\mathrm{F}$ es la frecuencia y $\mathrm{D}$ la dosis aplicada de biofertilizante, las dosis o concentración del biofertilizante comprende dosis de 0.5: 15 litros de agua y 0.25: 15 litros de agua. Con relación a la frecuencia de aplicación se realizó una cada 3 días, y la otra cada 5 días

El universo en estudio fue un área de $14^{*} 25$ metros con parcelas de 5 metros por 7 metros con una población de 100 plantas comprendidas en 5 surcos, lo cual define una población total del experimento de 800 plantas.

Las variables que se midieron son: desarrollo vegetativo, rendimiento productivo y ganancia neta. Las técnicas utilizadas fueron la observación y registro de campo donde se midieron cada uno de los indicadores una vez a la semana, el procesamiento de la información se realizó en INFOSTAT.

\section{Resultados y discusión}

La longitud de la guía del Cucumis sativa L. va desde la base de la planta hasta la última hoja, en este estudio se obtuvieron resultados que oscilan desde 146.12 hasta 170 centímetros como los muestra el siguiente gráfico. 


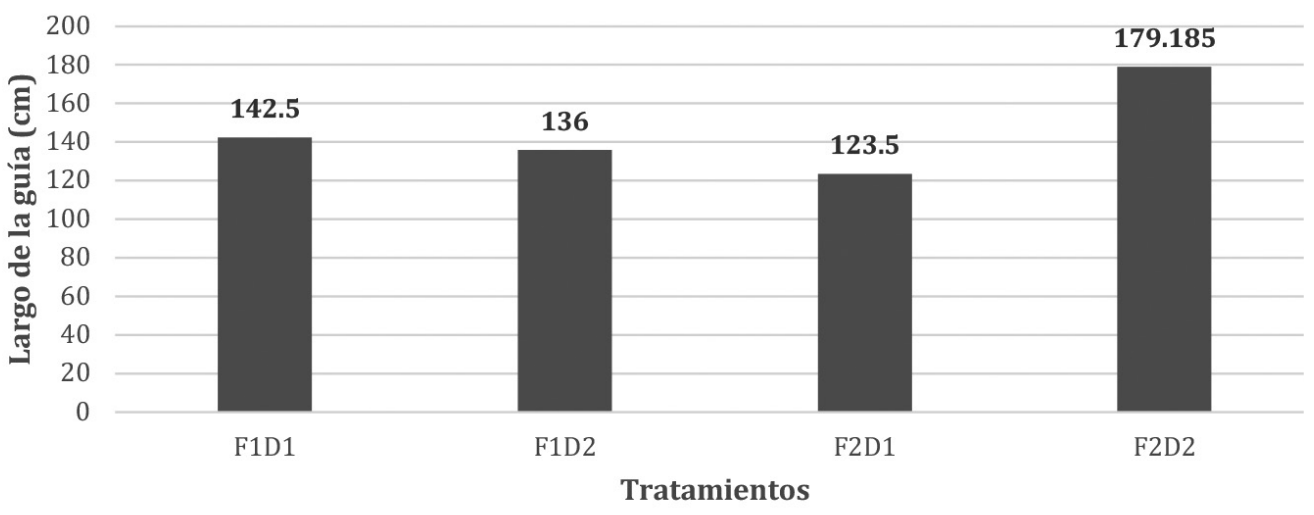

Gráfico 1. Longitud de la guía en cada uno de los tratamientos, Siuna.

Las longitudes de la guía del cultivo en cada uno de los tratamientos van desde los 127 a los 170 centímetros, siendo el tratamiento cuatro el que mejores resultados presenta lo cual coincide con Restrepo, (2000), el cual plantea que entre mayor sea la dosis y menor la frecuencia la planta no lo absorbe en un $100 \%$ por la alta concentración, además puede ser quemante.

Estadísticamente existen diferencias significativas al 5\% entre los tratamientos de concentración y frecuencias de aplicación de abonos orgánicos foliares (biofertilizante) en el largo de la guía del Cucumis sativa $L$, con un valor $\mathrm{p}=0,0004$ indicando las diferencias significativas. Lo que indica un $95 \%$ de confianza que al menos un tratamiento es mejor que los demás, siendo el tratamiento 4 de frecuencia de 5 días y dosis de 0.25 litros por bombada como se muestra en la tabla 1.

Tabla 1. Resultados de tratamientos, según análisis de diferencia mínima significativa de Fisher

\begin{tabular}{llll} 
Tratamientos & \multicolumn{2}{c}{ Medias n } \\
F2D1 & 123.50 & 2 & A \\
F1D2 $_{1}$ & 136.00 & 2 & B \\
$F_{1} D_{1}$ & 142.50 & 2 & $B$ \\
$F_{2} D_{2}$ & 179.19 & 2 & $C$
\end{tabular}

Letras distintas indican diferencias significativas $(\mathrm{p}<=0.05)$

Estos resultados coinciden con Marín (2007) el cual realizo estudios sobre la productividad del pepino con biofertilizantes indicando una altura o longitud de la guía de 176.9 centímetros, 3 por debajo de los alcanzado en esta investigación y difieren con Blandón, Blandón y Fernández, (2014). Donde con fertilización química solamente alcanzo resultados de 42.24 centímetros.

Con relación a la variable de número de frutos por planta los resultados oscilaron en un rango de 29 a 43 frutos, figura 2. 


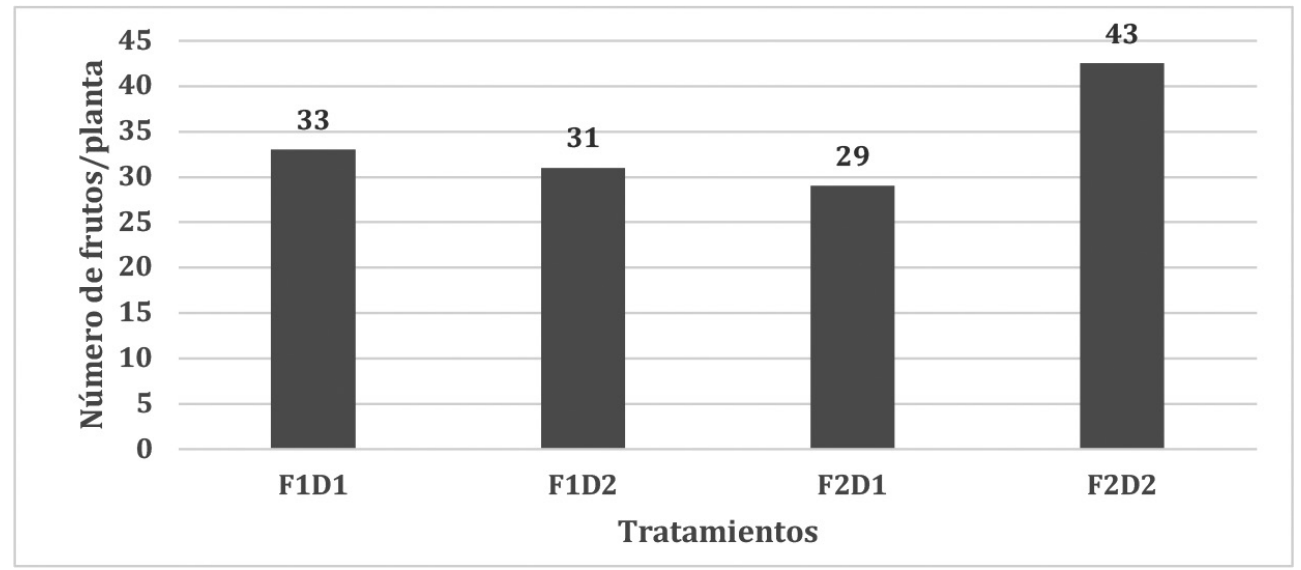

Figura 2. Numero de frutos por planta en cada tratamiento. Siuna

Los resultados encontrados en la variable número de fruto, estadísticamente hay diferencias significativas al 0.05 de probabilidad, con un valor $\mathrm{p}=0.0004$, menor que la significancia estimada, lo que indica que al menos uno de los tratamientos es mejor que los demás, lo cual implica un $48 \%$ de incremento, resultados que coinciden con López, Montano, Agustín, Beltrán y Berto, (2002) que indicaron hasta el $45 \%$ con concentraciones menores, esto se debe a que hay una mejor absorción en dosis más bajas pero más frecuentes.

La prueba de diferencia mínima significativa de Fisher demuestra el tratamiento que fue más efectivo en rendimiento fue el tratamiento 4 con una media de 43 con una diferencia de 10 frutos por planta que corresponde a un $30 \%$ más que el segundo tratamiento con mejores resultados.

$\begin{array}{lllllll}\text { Tratamientos } & \text { Medias n } & & & & \\ & & & & & \\ \text { F2D1 } & 26.50 & 2 & \mathrm{~A} & & \\ \mathrm{~F}_{1} \mathrm{D}_{2} & 29.00 & 2 & \mathrm{~A} & & \\ \mathrm{~F}_{1} \mathrm{D}_{1} & 33.00 & 2 & & \mathrm{~B} & \\ \mathrm{~F}_{2} \mathrm{D} 2 & 43.00 & 2 & & & \mathrm{C}\end{array}$

Letras distintas indican diferencias significativas $(\mathrm{p}<=0.05)$

En la variable de longitud del fruto los resultados oscilan entre 19 y 22 centímetros, figura 3. Los cuales coinciden con Marín (2017). En el indica resultados de 19 a 20,4 cm de longitud, con aplicaciones de foliares

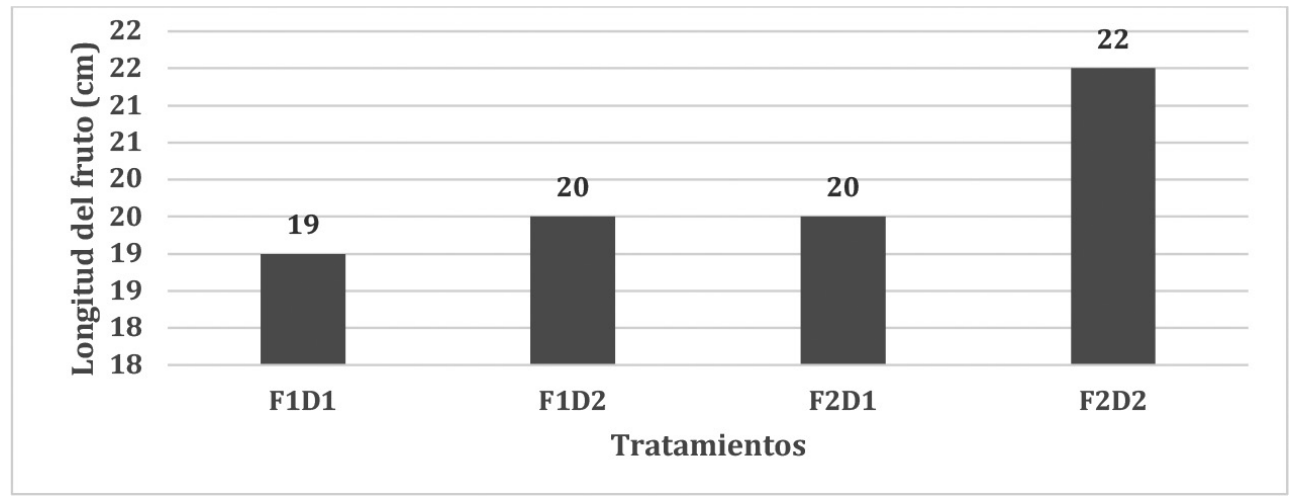

Figura 3. Longitud del fruto en el pepino con diferentes dosis y frecuencias de biofertilizantes. Siuna 
Estadísticamente no hay diferencias entre los tratamientos que se evaluaron, al $0.05 \%$ ya que el valor $\mathrm{p}$ $=0.8$, mayor que la significancia estimada, por lo que se comportó de manera similar, por lo que se asume que no hay incidencia de dosis ni frecuencias de aplicación del biofertilizante en el tamaño del fruto.

Con relación a la variable producción por hectárea, osciló una diferencia del $46.5 \%$ del tratamiento cuatro $\left(\mathrm{F}_{2} \mathrm{D}_{2}\right)$ con relación al tratamiento de menor rendimiento que fue el tres $\left(\mathrm{F}_{2} \mathrm{D}_{1}\right)$. Figura 4 . Las producciones de pepino obtenidas en este estudio difieren totalmente por lo planteado por agro negocios (2004) el cual establece que se producen 158,600 unidades de pepino mientras que los resultados de este estudio se obtuvieron como producción máxima 388,535 unidades por hectárea, 149\% más de incremento de la producción con la utilización de abonos orgánicos foliares (biofertilizantes con una frecuencia de 3 días y dosis de 0.25 litros en 15 litros de agua).

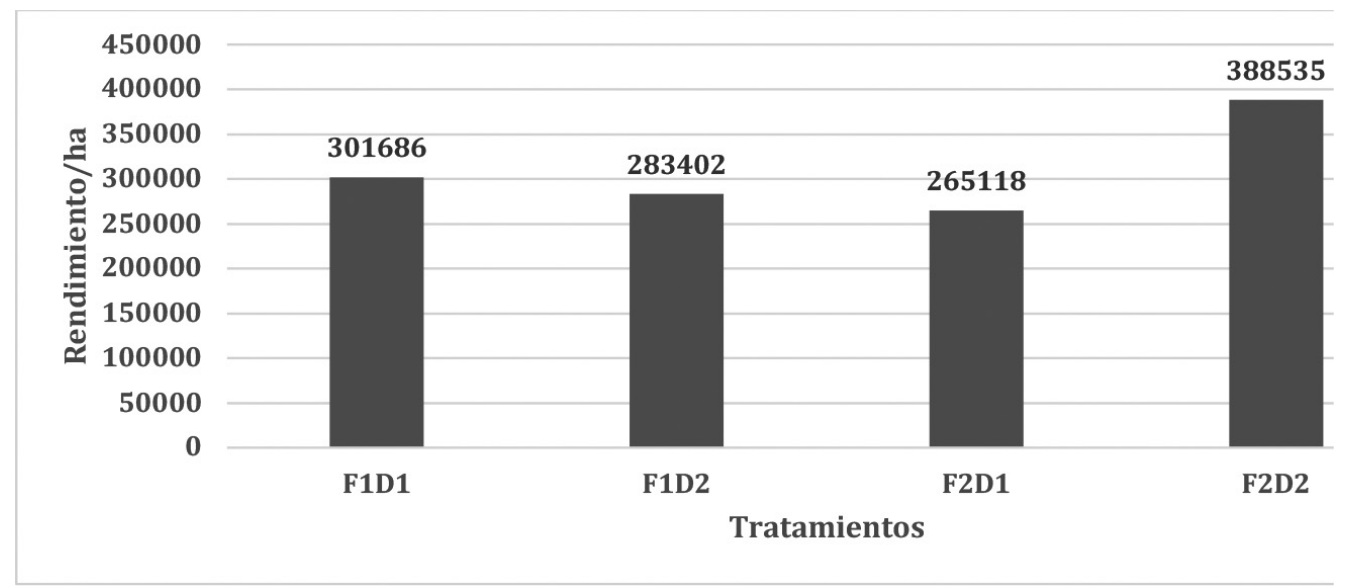

Figura 4. Rendimiento por hectárea del pepino, con diferentes dosis y frecuencias de biofertilizantes, Siuna

Los resultados de la variable de ganancia neta en esta investigación fueron de 370,251.00 córdobas de diferencias del tratamiento 4 con el tratamiento de menor ganancia, el cual fue el tratamiento 3 Tabla 1.

Tabla 1. Análisis económico de la produccion de pepino con diferentes dosis y frecuencias de biofertilizantes

\begin{tabular}{|l|l|l|l|l|l|}
\hline \multicolumn{1}{|c|}{ Descripción } & \multicolumn{1}{|c|}{ Costos/ha } & Producción/ha & \multicolumn{1}{|c|}{ Precio/unid } & \multicolumn{1}{c|}{$\begin{array}{c}\text { ingresos/ } \\
\text { ha }\end{array}$} & Ganancia neta \\
\hline Tratamiento 1 & $28,005.00$ & $301,686.00$ & 3 & $905,058.00$ & $877,053.00$ \\
\hline Tratamient0 2 & $23,095.00$ & $283,402.00$ & 3 & $850,206.00$ & $827,111.00$ \\
\hline Tratamiento 3 & $23,005.00$ & $265,118.00$ & 3 & $795,354.00$ & $772,349.00$ \\
\hline Tratamiento 4 & $24,845.00$ & $388,535.00$ & 3 & $1165,605.00$ & $1140,760.00$ \\
\hline
\end{tabular}

\section{Conclusiones}

El crecimiento y desarrollo en el cultivo del pepino son otras en los diferentes niveles de concentración y aplicación del fertilizante, con un valor $\mathrm{p}$ de 0.0004 por lo tanto estadísticamente existen diferencias significativas al 0.05 , siendo el tratamiento de frecuencia cada 5 días y dosis de 0.25 litros por bombada el mejor.

La producción de pepino es mayor en el tratamientos cuatro con un aproximado 388,535 de unidades un $46.5 \%$ más respecto al tratamiento tres, que obtuvo el segundo lugar en producción. 
Económicamente todos los tratamientos presentan un alto margen de ganancia neta; sin embargo, el que más margen presentó fue el tratamiento 4, obteniéndose una ganancia neta de 1165,605.00 córdobas netos equivalentes a 36425.15 dólares.

\section{Lista de referencias}

Altieri, M (1992). ¿Por qué estudiar la agricultura tradicional? Agro ecología y desarrollo. No.1.p.16-24.

Blandón, Z., Blandón, E., \& Fernández, N. (2014). Evaluación de fertilización orgánica y química en el rendimiento del cultivo de pepino (Cucumis sativus L,). Jinotega: UNAM LEON.

Castillo, J. \& Montalbán O. (200o). Comparación de la producción del cultivo del arroz de primera de forma tradicional y rebrote y aplicación de biofertlizantes, Siuna RAAN.

Centro de desarrollo de agro negocios (2004). Costos de producción del pepino. Boletín de producción \#54. Honduras

Cracogna, M., Fogar, M., Iglesias, M., \& Verón, R. (2001). Uso de lombricompuesto e inoculante con Azospirillum sp, en el cultivo del pepino (Cucumis sativus L.) en un suelo del Chaco. Argentina: UNNEN.

López Rivera R, Montano R, Agustín J., Beltrán V., Rodríguez Pérez Y., \& Berto Y. (2002). Evaluación de diferentes dosis de FitoMas (producto natural derivado de la caña de Azúcar), para determinar la dosis más efectiva en el cultivo del pepino, variedad SS- 5. Guantánamo Cuba.

Marín, N. (2017). Determinación de la productividad del pepino (Cucumis sativus L.) con vermicompost y biofertilizantes en invernadero. Torreón Coahuila: Universidad Autónoma Agragia Antonio Narro.

Restrepo J., (1996). La Idea y el arte de Fabricar los Abonos Verdes Fermentados. (Aportes y Recomendaciones). Una Experiencia de Agricultura en Centro América

Rodríguez, S. (2003). Comparación de cuatro variedades de arroz con y sin la aplicación de biofertilizantes, Siuna 2003.

Vásquez, E. (2012). Producción organica de pepino (Cucumis sativus L.) Con biofertilizantes y acolchado plástico en condiciones de casasombra. Saltillo, Coahuila, México. Recuperado el 12 de ocubre de 2018, de. http://repositorio.uaaan.mx:808o/xmlui/bitstream/handle/123456789/1512/ PRODUCCION\%20ORGANICA\%20DE\%20PEPINO\%20(Cucumis\%2osativus\%20L.)\%20CON\%20 BIOFERTILIZANTES\%20Y\%20ACOLCHADO\%20PLASTICO\%20EN\%20CONDICIONES\%20DE\%20 CASASOMBRA.pdf? sequence $=1$ 